ARTICLE

\title{
Structure and transport mechanism of P5B-ATPases
}

\author{
Ping Li, ${ }^{1,3}$, Kaituo Wang (1] 2,3, Nina Salustros ${ }^{2}$, Christina Grønberg ${ }^{2}$ \& Pontus Gourdon (10 1,2凶
}

In human cells, P5B-ATPases execute the active export of physiologically important polyamines such as spermine from lysosomes to the cytosol, a function linked to a palette of disorders. Yet, the overall shape of P5B-ATPases and the mechanisms of polyamine recognition, uptake and transport remain elusive. Here we describe a series of cryo-electron microscopy structures of a yeast homolog of human ATP13A2-5, Ypk9, determined at resolutions reaching $3.4 \AA$, and depicting three separate transport cycle intermediates, including spermine-bound conformations. Surprisingly, in the absence of cargo, Ypk9 rests in a phosphorylated conformation auto-inhibited by the $\mathrm{N}$-terminus. Spermine uptake is accomplished through an electronegative cleft lined by transmembrane segments 2, 4 and 6 . Despite the dramatically different nature of the transported cargo, these findings pinpoint shared principles of transport and regulation among the evolutionary related P4-, P5A- and P5B-ATPases. The data also provide a framework for analysis of associated maladies, such as Parkinson's disease.

\footnotetext{
${ }^{1}$ Department of Experimental Medical Science, Lund University, Lund, Sweden. ${ }^{2}$ Department of Biomedical Sciences, University of Copenhagen, Copenhagen N, Denmark. ${ }^{3}$ These authors contributed equally: Ping Li, Kaituo Wang. ${ }^{凶}$ email: pontus.gourdon@med.lu.se
} 
-type ATPases form a large membrane protein superfamily that couples ATP hydrolysis to the transport of cargo across biological membranes ${ }^{1}$. They are divided into five classes, P1-5, based on sequence similarity and transport specificity ${ }^{2}$. While P1-3 represent ion pumps prevalent in all kingdoms of life, P4-ATPases are phospholipid flippases that do not occur in prokaryotes. Analogously, P5-ATPases are ubiquitously present in eukaryotic species only, representing the least characterized class physiologically, mechanistically, and structurally. They are subdivided into two subclasses, P5A and P5B, based on the conservation of residues in the transmembrane domain ${ }^{3}$. The yeast P5A-ATPase Spf1 (hereafter referred to Spf1) was recently determined to be a helix dislocase required for the removal of mis-inserted hydrophobic helices from the ER membrane ${ }^{4}$. P5BATPases are more divergent, with typically four members per species $^{5}$, including human ATP13A2-5. ATP13A2 resides in the lysosome $^{6}$, its malfunction leads to ER-associated cell death ${ }^{7,8}$ and ATP13A2 mutations cause Kufor-Rakeb syndrome, a form of Parkinson's disease associated with dementia ${ }^{9,10}$. Equivalently, expression of ATP13A2 has been shown to protect against $\alpha$ synuclein-induced cytotoxicity ${ }^{11}$. Recently, ATP13A2 was demonstrated to transport physiological polyamines such as spermine (SPM) from the lysosomal lumen into the cytosol ${ }^{12}$. Together, these results advocate a fundamental role of P5BATPases in lysosomal health, thus representing attractive targets for studies and novel treatments of neurodegenerative diseases.

Biochemical data suggest that P5B-ATPases comprise the conserved P-type ATPase core, with three cytoplasmic domains and a transmembrane domain similar to P2-, P3-, and P4ATPases. However, P5-ATPases feature a unique N-terminus, with two additional TM helices, Ma and Mb, in P5A-ATPases, distantly reminiscent to the $\mathrm{P} 1 \mathrm{~B}-\mathrm{ATPases}$, which are predicted to be absent in $\mathrm{P} 5 \mathrm{Bs}^{5,13,14}$. Thus, even the topology, overall architecture, and domain organization of P5Bs remain elusive. P5ATPases are believed to exploit the classical P-type ATPase reaction cycle, with alternating access between inward-facing E1 and outward-facing E2 states, linked to autophosphorylation and dephosphorylation, respectively, through four principal states, E1-E1P-E2P-E2 (P denotes phosphorylated configurations) (Fig. 1a) ${ }^{15,16}$. Here, we provide a series of single-particle cryo-electron microscopy (cryo-EM) structures of the P5BATPase Ypk9 from the thermophilic yeast Chaetomium thermophilum (hereafter denoted Ypk9), granting new structural insights into transport and regulation mechanisms of P5B-ATPases.

\section{Results and discussion}

Overall structure and conformation. Ypk9 was initially studied using nanodisc-reconstituted C-terminally green fluorescent protein (GFP)-fused sample. A cryo-EM structure, coined E2Pinhib, was determined at an average resolution of $3.5 \AA$, generated in the presence of the phosphate analog beryllium fluoride $\left(\mathrm{BeF}_{3}{ }^{-}\right)$, which has been previously employed as a conformationstabilizing tool for investigations of P-type ATPases ${ }^{17}$ (Methods and Supplementary Table 1). The cryo-EM maps show wellresolved domains (Supplementary Figs. 1a and 2), enabling de novo model building of the entire ATPase, except for certain peripheral loops of the soluble domains, and some $200 \mathrm{~N}$ terminal residues that are largely absent in the human P5BATPase members (Supplementary Fig. 3). The structure reveals a typical P-type ATPase fold, including the conserved cytosolic actuator (A-), phosphorylation (P-), and nucleotide-binding (N-) domains, and a transmembrane (M-) domain composed of ten transmembrane spanning helices, TM1-10 (Fig. 1). In addition, an N-terminal domain (NTD) structurally related to the one observed for the yeast P5A-ATPase Spf1 is detected ${ }^{4}$ (Supplementary Fig. 4). Nevertheless, comparisons to the Spf1 structures reveal major differences, such as a unique NTD topology (see further below), and lack of the so-called arm domain, an $\alpha$-helical elongation emerging from the P-domain of Spf1 ${ }^{4}$. Furthermore, weak cryo-EM density is present immediately adjacent to the cytosolic leaflet, located between the first assigned residue of the NTD and the P-domain, though no secondary structure features are visible. The presence of an elongated $\mathrm{N}$-terminus, prior to the NTD, appears unique to certain P5B-ATPases, as such extensions are absent in the P5A subclass (Supplementary Figs. 3 and 4). Structure-based alignments of the soluble A-, N-, and P-domains to different structures of the well-studied P2 sarco/ endoplasmic reticulum $\mathrm{Ca}^{2+}$-ATPase (SERCA) and the P4ATPase ATP11C-CDC50A ${ }^{18}$ suggest that our structure resembles
A

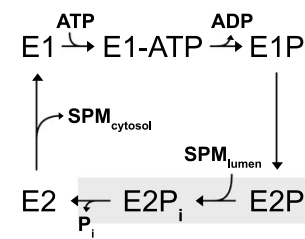

C

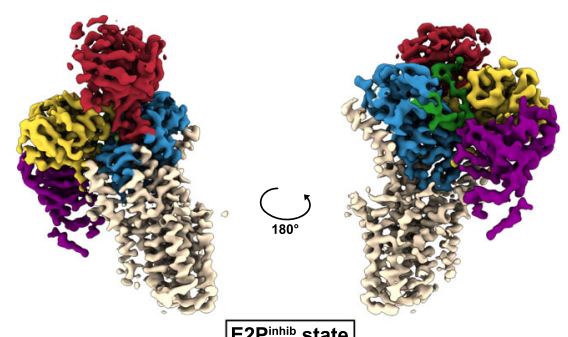

E2Pinhib state
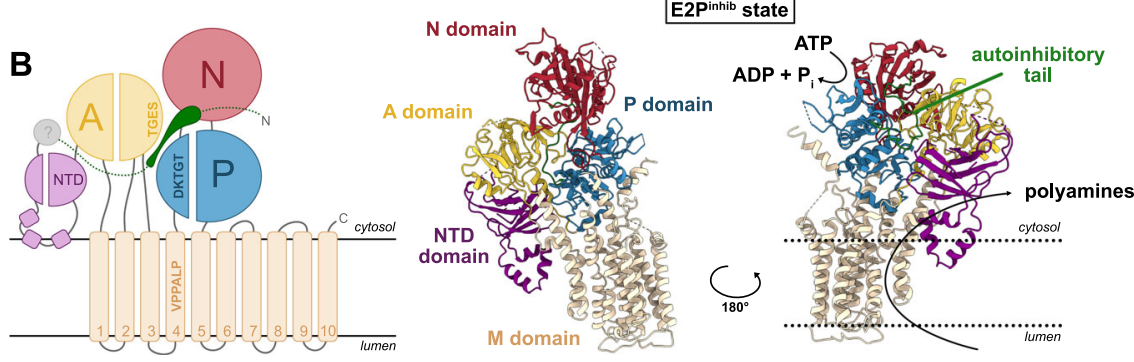

Fig. 1 Overall P5B-ATPase structure and topology. a The P5B-ATPase transport cycle likely follows the E1-E1P-E2P-E2 Post-Albers scheme shown here, with the part of the focus in this work highlighted in gray. ATP consumption energizes the transport of polyamines such as spermine (SPM). b The P5B-ATPase topology, with ten transmembrane helices TM1-10 and the cytosolic A-, P-, and N-domains are conserved among P-type ATPases. The P5-specific N-terminal domain (NTD, pink) harbors three short helices dipping into the membrane. Moreover, N-terminal residues form an autoinhibitory tail (green) interacting with the $\mathrm{A}-, \mathrm{P}-$, and $\mathrm{N}$-domains in the E2Pinhib state. c EM density and cartoon representation of the E2Pinhib conformation with contour level $=0.8$. 

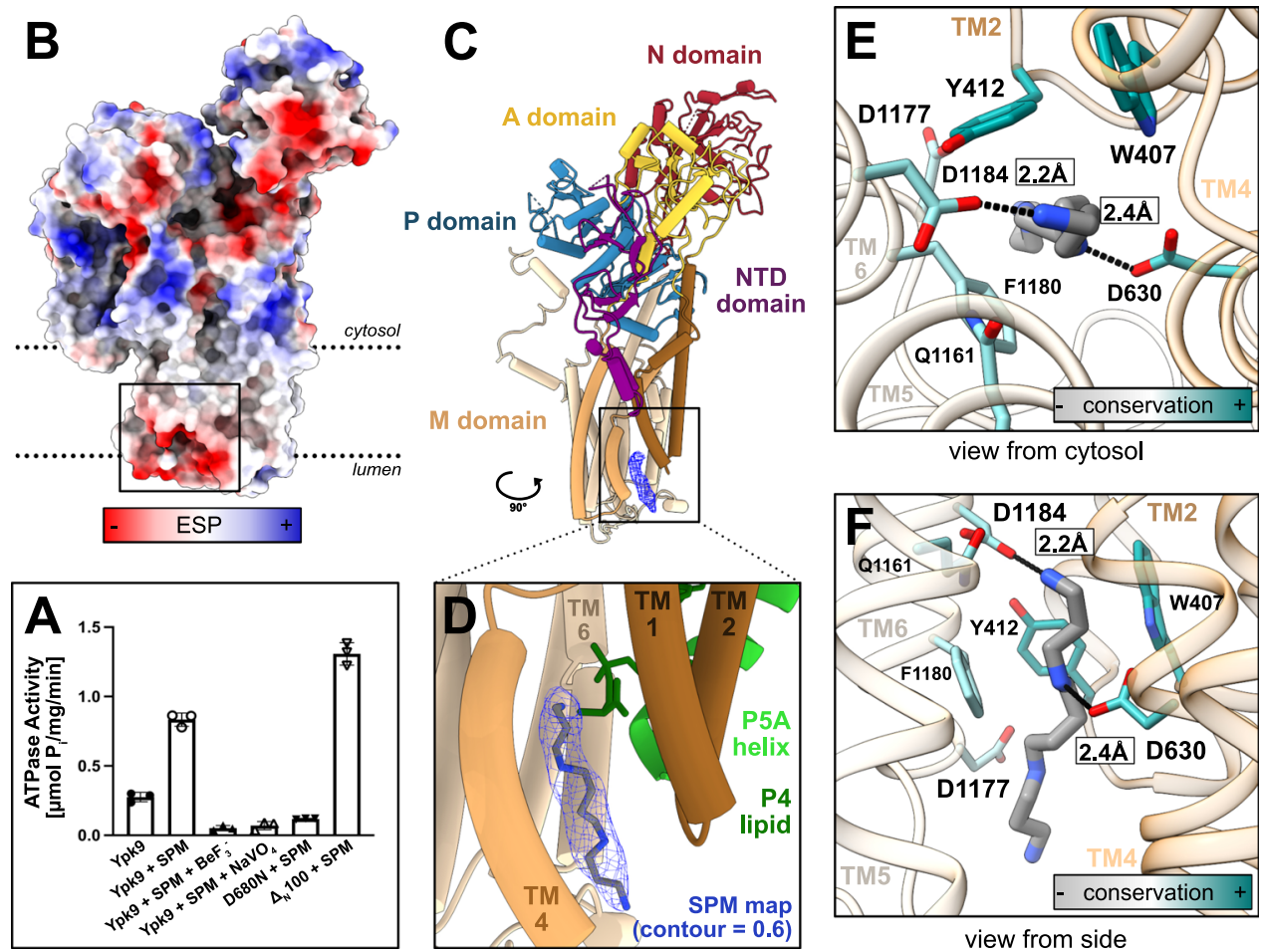

Fig. 2 Polyamine uptake. a ATPase activity of purified Ypk9 is stimulated by the polyamine spermine (SPM) and inhibited by the inhibitors BeF ${ }_{3}^{-}$and $\mathrm{NaVO}_{4}{ }^{-}(n=3$ independent measurements, error bars represent the mean with SD). The function is abolished by a classical P-type ATPase dead-mutation of the catalytic aspartate $(D 680 N)$, and instead stimulated when the first 100 amino acids are removed $\left(\Delta_{N} 100\right)$. $\mathbf{b}$ Surface electrostatics of the E2.P state. The luminal surface is highly electronegative, congruent with polyamine access. c, d Side-view and close-up of the SMP binding pocket. d Alignment of the E2.P $P_{i}$ state and the cargo-bound structures of the P5A-ATPase Spf1 (PDB-ID 6XMU) and the P4-ATPase ATP8A1-CDC50 (6K7M), showing only their helix and lipid in green. SPM density is displayed as blue mesh, map contour $=0.6$. e, $\mathbf{f}$ Close-view of the SPM binding pocket and coordinating residues. Side chains are colored by conservation, as determined by AL2CO implemented in ChimeraX 42,45 , excluding side chains with poor conservation (<0.5).

an outward-facing E2P state, congruent with the open configuration caught by $\mathrm{BeF}_{3}{ }^{-}$in other P-type ATPases (Supplementary Fig. 5 and Supplementary Table 2). As an example, the conserved A-domain TGES dephosphorylation loop is located near the catalytic aspartate. Considering that the E2P state is known to be linked to counter cargo uptake from the outside in ion-transporting P-type ATPases, the obtained state is presumably linked to polyamine recognition in P5B-ATPases, an enigmatic aspect of these proteins.

Spermine uptake and transport. The direct transport of the biogenic polyamines SPM and spermidine (SPD) from the lysosome to the cell interior by the human P5B-ATPase ATP13A2 has recently been demonstrated ${ }^{12}$. To unravel the underlying molecular transport mechanism, we first confirmed SPMstimulated activity for Ypk9 in vitro, using a dead-mutant of the catalytical phosphorylation site, D680N, as a control (Fig. 2a). Next, additional structures were determined, all in the presence of SPM, and using N- or C-terminally linked GFP protein. We exploited conditions in the presence again of $\mathrm{BeF}_{3}{ }^{-}$(yielding a structure at $3.7 \AA$ resolution overall, denoted E2P*), or without a similar compound ( $3.5 \AA$, E2.P. $\mathrm{P}_{\mathrm{i}}^{\mathrm{SPM}}$ ) (Supplementary Table 1, Supplementary Figs. 1a, $\mathrm{b}$ and 2). Moreover, the alternative phosphate mimic aluminum fluoride, $\mathrm{AlF}_{4}{ }^{-}$, was applied $(3.4 \AA$,

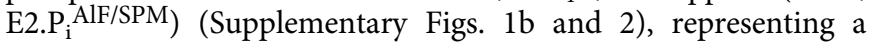
compound known to catch a transition state of dephosphorylation in-between E2P and E2, E2.P $\mathrm{P}_{\mathrm{i}}$, which is cargooccluded in ion-transporting P-type ATPases ${ }^{19}$. Overall, the different SPM data collected show well-resolved soluble domains, although the $\mathrm{N}$-domain density is somewhat poor (Supplementary Fig. 1b). Structural analyses to SERCA and
ATP11C-CDC50A suggest the SPM-structures are indeed caught in separate E2P and E2.P $P_{i}$ configurations, with the E2.P $\mathrm{P}_{i}^{\mathrm{SPM}}$ structure indicating the presence of cargo is sufficient to stabilize an E2.P $P_{i}$ state (Supplementary Table 2). While the E2.P $P_{i}$ structures are highly similar, distinctions are identified between the two recovered E2P states (Supplementary Fig. 6, see further below).

Interestingly, the E2.P $\mathrm{P}_{\mathrm{i}}$ models exhibit a highly electronegative luminal surface (Fig. 2b and Supplementary Fig. 7), from which an opening emerges between TM2, 4, and 6 , reaching the central TM4 kink of all P-type ATPases, and the VPPALP P5B-signature motif. The cleft harbors an additional, elongated density compatible with the presence of SPM (Fig. 2c, d). The pocket is permitted by an unwound TM4 and is formed by several electronegative and hydrophobic side chains, of which some, i.e., the P5B-conserved D630 in TM4, Y1157 in TM5, D1177, and D1184 in TM6, appear directly interacting with SPM (Fig. 2e, f, Supplementary Figs. 3 and 8). Indeed, in vitro studies on the D962N variant of ATP13A2 (D1184N in Ypk9) revealed a complete loss of ATPase activity and spermine-induced dephosphorylation ${ }^{12}$, highlighting the central role of this residue in polyamine detection and transport. As such, the composition of the cavity also allows binding of shorter physiological polyamines, e.g., SPD and putrescine, in a similar manner. Moreover, we note that the TM2, 4, and 6 cargo-binding area overlaps well with the one observed in both P5A-ATPases, Spf1 (PDB-ID 6XMU), and P4-ATPases, ATP8A1-CDC50a (6K7M) and ATP11C-CDC50a (6LKN and 7BSU) (Fig. 2d) $4,18,20,21$, and that these separate cargoes are less occluded as compared to P2and P3-ATPases. Thus, it appears that critical features of the transport pathway are conserved independent of lipid, helix, or 


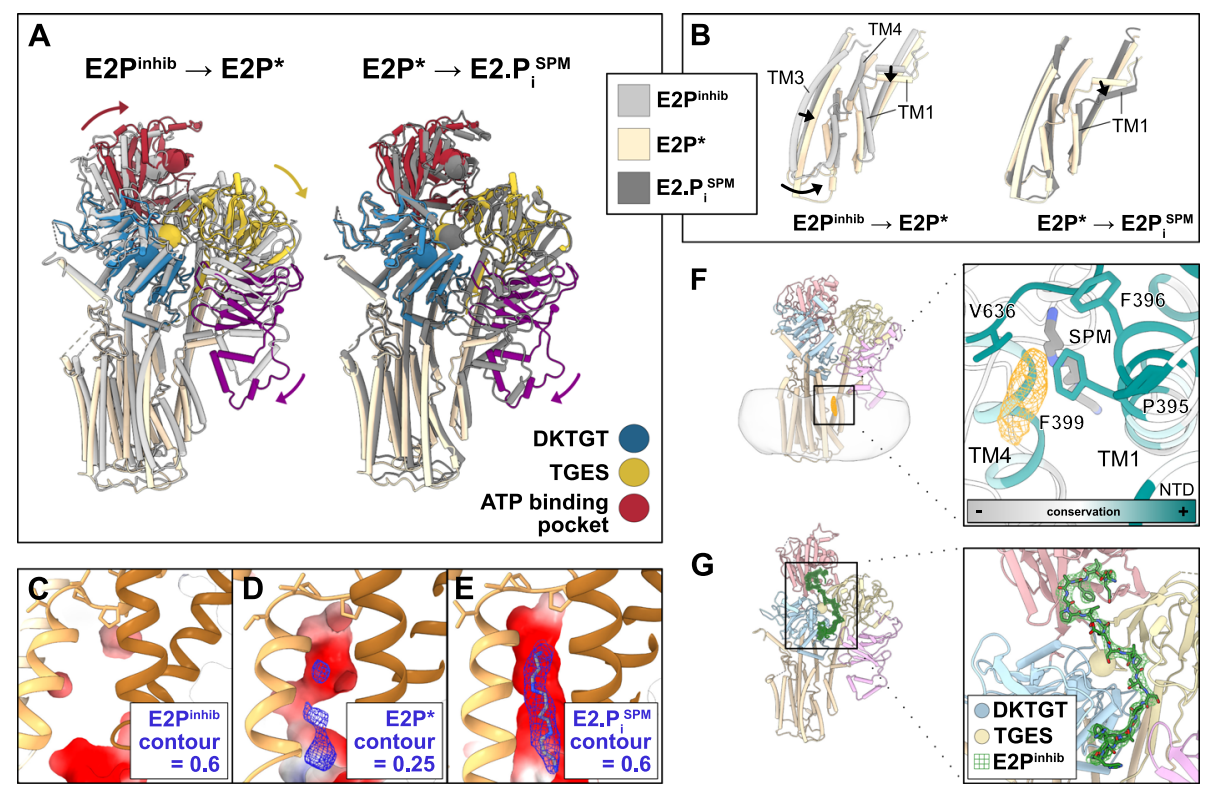

Fig. 3 Conformational changes during polyamine uptake, lipid regulation, and auto-inhibition. a Alignment of the generated structures on the $\mathrm{P}$ domain. Conserved motifs in the soluble domains are shown as spheres. b Alignment on TM5-10, and close-view of conformational changes in TM1-6. c-e Closeview of the polyamine binding pocket in the E2Pinhib $(\mathbf{c}), E 2 P^{\star}(\mathbf{d})$, and E2.P $\mathrm{P}_{\mathrm{i}}(\mathbf{e})$ states. EM density around SPM is shown in the blue mesh. $\mathbf{f}$ Side-view and a close-up of the lipid density in a conserved cleft formed by TM1,3, and 4, illustrating the position of the lipid at the cytosolic membrane leaflet. The closeview shows conserved side chains in proximity to the lipid density, colored by conservation, as determined by AL2CO implemented in ChimeraX 42,45 , excluding side chains with poor conservation $(<1)$. $\mathbf{g}$ Side- and close-views showing the location of the auto-inhibitory $\mathrm{N}$-terminal residues (mesh, dark green) in the E2Pinhib state. The DKTGT and TGES motifs of the P- and A-domains are shown as spheres. The autoinhibitory residues are interacting with the TGES loop and the nucleotide-binding pocket of the $\mathrm{N}$ domain.

polyamine cargo, in agreement with the shared common evolutionary origin of P4- and P5-ATPases ${ }^{5}$. Nevertheless, details of the mechanistic principles differ in-between the subclasses, i.e., SPM appears more protein-buried than the helix of P5AATPases, and yet solvent-exposed to the luminal side, similar to the lipid headgroups in $\mathrm{BeF}_{3}{ }^{-}$-stabilized conformations of $\mathrm{P} 4$ ATPases ${ }^{18,20}$.

Comparison of the structures reveals major rearrangements of the TM helices linked to SPM uptake (Fig. 3a, b; Supplementary Figs. 7 and 8). The electronegative luminal surface is present in all structures (Supplementary Fig. 7). However, while we do not detect polyamine indications in the E2Pinhib structure, poor cryoEM density for SPM, somewhat shifted toward the luminal side compared to the E2.P $\mathrm{P}_{\mathrm{i}}$ structures are available for E2P* (Fig. $3 \mathrm{c}-\mathrm{e}$ ).

The SPM binding pocket is not yet defined in the E2Pinhib conformation, where the luminal parts of TM1-2, TM3-4, and TM5-10 are further apart. Based on these observations, we speculate that the affinity for SPM increases from the E2Pinhib to the E2P* and E2.P $\mathrm{P}_{\mathrm{i}}$ states. The structural changes that orchestrate the SPM pocket are coupled to a rotation of the A-domain, leading to a $6 \AA$ movement of the TGES dephosphorylation loop as observed in other P-type ATPases, and a similar movement of the adjacent NTD (Fig. 3a).

A Ma-Mb depleted NTD. P5A-ATPases feature two extra TM helices, $\mathrm{Ma}$ and $\mathrm{Mb}$, emerging as an insert to the NTD. However, previous biochemical studies on $\mathrm{P} 5 \mathrm{~B}$-ATPases have indicated a topology with ten transmembrane helices only, and yet NTDlinked lipid-regulation ${ }^{5,14}$, leaving the assembly and mechanistic role of the $\mathrm{N}$-terminus of P5A-ATPases elusive. While peripheral parts are somewhat discontinuous in the densities, our structures clearly demonstrate that $\mathrm{Ma}$ and $\mathrm{Mb}$ are absent in P5B-ATPases (Fig. 1b, c). Instead, an amphipathic loop is formed by three $\alpha$ helices likely embedded in the membrane interface. Several of the residues in this stretch are highly preserved, including the hydrophobic L246 and W250, which may serve as a lipid bilayer anchor, as well as helix-terminating G242 (Supplementary Fig. 3). However, the remaining part of the NTD, essentially composed of a seven-stranded $\beta$-barrel directly linked to the A-domain, superposes well with the P5A structure (Supplementary Fig. 4), suggesting a common origin of the NTD for all P5-ATPases.

What is then the functional role of the NTD? Notably, in the membrane of the E2. $P_{i}$ structures, a single strong additional density not explained by the protein or SPM is located in a cleft formed by transmembrane helices TM1, 3, and 4 (Fig. 3f). The feature is in close proximity $(<6 \AA)$ to the conserved V636 of the VPPALP signature motif of TM4, but also the membraneembedded loop of the NTD. Previous studies have demonstrated a direct interaction of both phosphatidic acid (PA) and phosphatidylinositol $(3,5)$ bisphosphate $(\mathrm{PI}(3,5) \mathrm{P} 2)$ with three separate stretches of the ATP13A2 NTD and a stimulatory effect on ATPase activity ${ }^{14}$. Interestingly, two of these NTD regions are located in the membrane-dipping region of the Ypk9 NTD (L248-P252 and K257-K262), and the remaining portion is in close proximity to the observed density. We, therefore, hypothesize that the observed density relates to a lipid of the cytosolic membrane leaflet. The lipid may serve a regulatory function, linking the NTD and A-domain to TM1, 3, and 4. However, the presence of an unpredicted glycosylation site or even a non-native detergent molecule cannot be excluded (alternative lipid effects via the highly electropositive region around the C-terminus are also possible, Supplementary Fig. 9). The location close to the SPM-interacting 'spine' M4 transmembrane helix of P-type ATPases may permit coupled binding of the lipid and cargo in P5B-ATPases. Indeed, the NTD-domain is displaced between the E2P and E2. $P_{i}$ structures (Fig. 3a). We also note that the topological differences of P5A- and P5B-ATPases in this way may represent two separate principles of conferring lipid-anchored NTD-influence to the ATPase core. 


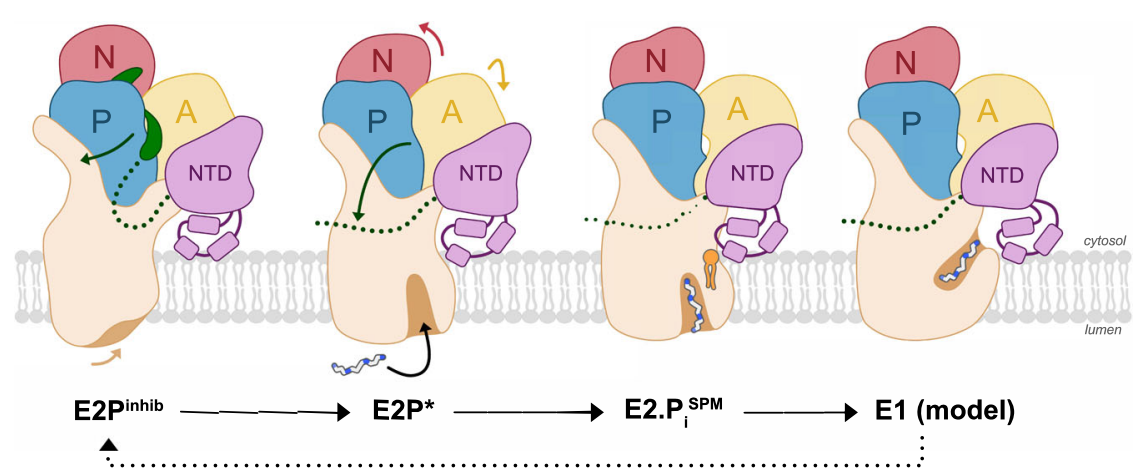

Fig. 4 Proposed transport mechanism. We suggest P5B-ATPases rest in a phosphorylated state, auto-inhibited through interactions of $\mathrm{N}$-terminal residues with the cytosolic $\mathrm{A}-, \mathrm{P}-$, and $\mathrm{N}$-domains (E2Pinhib). Polyamine binding and the coupled dephosphorylation lead to the release of the autoinhibitory loop, and the formation of a binding pocket between TM 2, 4, and 6 (E2 $\left.\mathrm{P}^{\star}\right)$. It is possible that the formation of the cavity and polyamine uptake may be stimulated through interactions of the NTD with a regulatory lipid (E2.P.PPM, orange). Dephosphorylation and the subsequent E2 $\rightarrow$ E1 transition will then lead to cargo release to the cytosol, priming the ATPase for nucleotide binding, phosphorylation, and a new transport cycle.

Auto-inhibition. The E2P structures exhibit another peculiar feature. Sandwiched in-between the soluble domains, a long continuous density is observed. While poorly resolved in the E2P* data, we assign the stretch to residues D74-G97 of the Ypk9 $\mathrm{N}$-terminus based on visible side chains in the E2Pinhib cryo-EM density (Fig. 3g). Strikingly, it interacts with several key aspects for ATP-hydrolysis and auto-dephosphorylation which are omnipresent among P-type ATPases, namely the TGES dephosphorylation motif of the A-domain, the nucleotide-binding pocket in the $\mathrm{N}$-domain, and parts of the surface of the $\mathrm{P}$ domain $^{22}$. The C-terminus of the loop is also in close proximity to the above-mentioned poorly resolved density that likely bridges to the NTD (Supplementary Fig. 10).

While such properties have not been assigned to P5-ATPases previously to our knowledge, this observation brings to mind the regulatory termini known to be present in many P-type ATPase subclasses, including P1B, P2B, P3A, and P4-ATPases ${ }^{23-26}$. In the more $\mathrm{P} 5$-alike $\mathrm{P} 4$ class, auto-inhibitory $\mathrm{C}$-terminal domains have been detected in both human ATP8A1-CDC50a and yeast Drs2p-Cdc50p, tightly blocking the nucleotide-binding pocket ${ }^{18,27}$. As the loop was exclusively observed in a $\mathrm{BeF}_{3}{ }^{--}$ stabilized E2P conformation, it was suggested to specifically stabilize this particular state of P4-ATPases ${ }^{18}$. A comparison of the $\mathrm{N}$-terminal loop in $\mathrm{Ypk} 9$ with the $\mathrm{C}$-terminal regulatory domain of P4-ATPases exposes distinct similarities, such as the interference with the nucleotide-binding pocket and binding of the stretch in the E2P but not the E2.P ${ }_{\mathrm{i}}$ states (Supplementary Fig. 10). Indeed, a Ypk9 form lacking the first 100 residues, Ypk9 $\Delta_{\mathrm{N}} 100$, also demonstrates higher activity in our in vitro assay (Fig. 2a). Thus, it appears as if the observed N-terminal feature indeed serves an auto-inhibitory role, perhaps exclusively stabilizing the E2P conformation, and that the P4- and P5BATPase regulatory mechanisms share common features. The aforementioned notion is not in disagreement with the observed "resting" autophosphorylated configuration detected in ATP13A2 ${ }^{14}$. This is because the N-termini of P5B-ATPases are not conserved (Supplementary Figs. 3 and 4), and such a mechanism would likely be limited to P5B-ATPases with an $\mathrm{N}$ terminus of sufficient length, e.g., Ypk9. Also, complementary biochemical data are needed to confirm the proposed autoinhibition mechanism.

Nevertheless, these inhibitory P4- and P5B-loops also display unique aspects, such as that they emerge from different termini. In this light, the configuration of the C-terminus, following the last transmembrane helix, TM10, is of interest. A cytosolic extension is indeed present also in Ypk9, and while the preceding membrane-spanning part superposes well with other P-type
ATPases, the soluble portion remains helical but is kinked near the conserved E1351 (Supplementary Fig. 9). Next, the Ypk9 Cterminus forms an additional small, soluble helix, which directly interacts with the $\mathrm{P}$-domain, compatible with regulatory properties as it may stabilize the relative organization of the P- and TMdomains. However, the localization of the C-terminus is maintained in all our structures, including constructs with $\mathrm{N}$ and C-terminal GFP-fusions, hence excluding conformational bias caused by construct design, rendering it difficult to unequivocally assign a critical functional role also to the Cterminus of P5B-ATPases.

Proposed transport mechanism. P5B-ATPases have previously been suggested to follow the conserved Post-Albers reaction cycle of P-type ATPases ${ }^{15,16}$. Presumably, E1 to E1P autophosphorylation of the catalytic aspartate occurs in a polyamine-independent manner ${ }^{14}$. Although this contrasts to ion-transporting P1-3-type ATPases, this is similar to P4-ATPases ${ }^{26}$, again highlighting the common evolutionary origin of these subfamilies. In the E2Pinhib structure following E1P, the N-terminus is likely auto-inhibiting the pump through interference with the soluble A-, $\mathrm{P}-$, and $\mathrm{N}$-domains, thereby preventing turn-over (Fig. 4).

Formation of the cargo-binding pocket due to shifts of TM3 and 4 is likely via TM1, 2, and 3 coupled to major rearrangements of the soluble domains, in particular displacements of the A- and NTD-domains. As observed in our E2P* structure, these shifts weaken the interactions between the $\mathrm{N}$-terminal loop and the ATPase core, leading to dissociation of the auto-inhibitory stretch from the soluble A-, P-, and N-domains. Simultaneously, indications of SPM between TM2, 4, and 6 are detected, and we denote this state as E2P*, as dephosphorylation has been initiated. The structural rearrangements may be reinforced through binding of regulatory lipids, possibly between TM1, 3, and 4 (as observed in our E2.P ${ }_{i}{ }^{S P M}$ structure), which is sensed by the NTD, thereby facilitating rotation of the NTD/A-domain. This agrees with the previously reported stimulatory effect of PA and $\mathrm{PI}(3,5) \mathrm{P} 2$ on ATP13A2 turnover, which was abolished through mutations in the membrane-dipping loop of the $\mathrm{NTD}^{14}$. However, the release of the auto-inhibitory loop could as well be achieved through interactions with regulatory lipids and/or auxiliary proteins, as observed for the yeast P4-ATPase Drs2 28,29 , or phosphorylation, as for human ATP8A2 $2^{30}$ Equivalently, completion of cargo-binding is linked to the proceeding E2.P $\mathrm{i}_{\mathrm{i}}$ state, where SPM is accommodated in a cavity on the luminal side, coordinated by conserved acidic and hydrophobic residues, including the conserved VPPALP motif. 
Cargo transport and release to the cytosol is then likely accomplished following dephosphorylation, in the E2 to E1 transition. We propose the exit pathway is formed by M2, 4, and 6, similar to the mechanism assumed for P4- and P5AATPases ${ }^{4,18,20}$. The absence of conserved and/or electronegative residues in this cleft suggests a direct release of the cargo from the observed high-affinity SPM binding site to the cytosol. Alternatively, due to the conserved nature of TM1 among P5BATPases, the exit pathway may be composed of TM1, 3, and 4, similar to the ion-uptake pathway in P1-3-type ATPases. This would, however, require a rather complex release mechanism, including re-arrangement of TM4 relative to TM1 to permit the polyamine to reach TM1, and we, therefore, believe that TM1 fulfills a role in lipid regulation rather than in cargo release.

The structures of Ypk9 presented here provide novel insights into the overall architecture and polyamine transport mechanism of P5B-ATPases. Ypk9 exhibits ten TM helices and a soluble Nterminal domain (NTD) in close proximity to the membrane, perhaps involved in lipid regulation. An N-terminal autoinhibitory loop likely stabilizes the $\mathrm{E} 2 \mathrm{P}$ state, regulating polyamine turnover. Spermine uptake occurs from the luminal side, through a conserved electronegative pocket formed by TM2, 4, and 6 . It is expected that conformational changes upon dephosphorylation trigger cargo translocation to the cytosol. However, additional structural and functional characterization is essential to fully elucidate the P5B-ATPase transport mechanism.

\section{Methods}

Cloning, overproduction, and purification of Ypk9. Full-length Ypk9 (Uniprot G0S7G9) gene was amplified using polymerase chain reaction with Phusion " HighFidelity DNA Polymerase (ThermoFisher scientific) from genomic DNA of $C$. thermophilum (DSM1495) using the primers ctP5B-22b-F, ctP5B-22b-R (Supplementary Table 3), and then cloned into the pET-22b vector using the NEBuilder HiFi DNA Assembly Master Mix. The seven introns were individually removed using $\Delta$ intron $1-F / \Delta$ intron1-R, $\Delta$ intron2-F/ $\Delta$ intron2-R, $\Delta$ intron1-2/ $\Delta$ intron2-R, $\Delta$ intron3-F/ $\Delta$ intron3-R, $\Delta$ intron4-F/ $\Delta$ intron4-R, $\Delta$ intron5-F/ $\Delta$ intron5-R, $\Delta$ intron6-F/ $\Delta$ intron6-R, and $\Delta$ intron7-F/ $\Delta$ intron7-R primer pairs listed in Supplementary Table 3. The ctP5B-N-GFP-F/ctP5B-N-GFP-R and ctP5B-C-GFP-F/ ctP5B-C-GFP-R primer pairs were used for fusion with $\mathrm{N}$ - and C-terminal GFP, respectively. The $\Delta$ N100-F/ctP5B-C-GFP-R primer pair was used for generating the $\Delta \mathrm{N} 100$ deletion form. The gene was confirmed by sequencing using Macrogen Europe. A TEV cleavage site, ENLYFQ, GGGGS linker, and green fluorescence protein (GFP) with a 10xHis tag were fused to the N- $\left(\mathrm{E} 2 \mathrm{P}^{*}\right.$ and E2.P. $\mathrm{P}_{\mathrm{i}}^{\mathrm{ALF} / \mathrm{SPM}}$ structures) or C-terminus (E2Pinhib and E2.P $\mathrm{P}_{\mathrm{i}}^{\mathrm{SPM}}$ ) of $\mathrm{Ypk} 9$, and cloned into the pEMBLyex4 expression vector ${ }^{31}$. The expression plasmids pEMBLyex4-Ypk9TEV-G4S-GFP-His ${ }_{10}$ and pEMBLyex4-10xHis ${ }_{10}$-GFP-G4S-TEV-Ypk9 were separately transformed into the PAP1500 Saccharomyces cerevisiae strain ${ }^{31}$ using the LiAc/SS carrier DNA/PEG method ${ }^{32}$ and plated on SD agar plates $(1.9 \mathrm{~g} / \mathrm{L}$ Yeast Nitrogen Base, $5 \mathrm{~g} / \mathrm{L}$ Ammonium sulfate, $20 \mathrm{~g} / \mathrm{L}$ Glucose, $30 \mathrm{mg} / \mathrm{L} \mathrm{Lys}, 60 \mathrm{mg} / \mathrm{L}$ Leu, $1.5 \%(\mathrm{w} / \mathrm{v})$ Agar).

Protein production was accomplished according to previously reported protocols $s^{31}$ with small modifications. Briefly, single colonies from freshly transformed plates were inoculated in $5 \mathrm{~mL}$ synthetic minimal (SD) medium supplemented with leucine (Leu) $(60 \mathrm{mg} / \mathrm{L})$ and lysine (Lys) $(30 \mathrm{mg} / \mathrm{L})$ at $30^{\circ} \mathrm{C}$ with $220 \mathrm{rpm}$ shaking for $24 \mathrm{~h}$. $2 \mathrm{~mL}$ overnight preculture was diluted in $100 \mathrm{~mL}$ $\mathrm{SD}$ media ( $1.9 \mathrm{~g} / \mathrm{L}$ yeast nitrogen base, $5 \mathrm{~g} / \mathrm{L}$ ammonium sulfate, $20 \mathrm{~g} / \mathrm{L}$ glucose) supplied with $30 \mathrm{mg} / \mathrm{mL}$ Lys and cultivated at $30^{\circ} \mathrm{C}$ with $200 \mathrm{rpm}$ shaking for around $30 \mathrm{~h}$. The cells were pelleted and transferred to $800 \mathrm{~mL}$ expression media $(1.9 \mathrm{~g} / \mathrm{L}$ yeast nitrogen base, $5 \mathrm{~g} / \mathrm{L}$ ammonium sulfate, $5 \mathrm{~g} / \mathrm{L}$ glucose, $1.1 \mathrm{~g} / \mathrm{L}$-Ile,Ura dropout amino acid mixture, $3 \%(\mathrm{v} / \mathrm{v})$ glycerol) at $30^{\circ} \mathrm{C}$ for $20 \mathrm{~h}$. Protein production was induced through the addition of $2 \%(\mathrm{w} / \mathrm{v})$ galactose dissolved in expression media lacking glucose, and protein production was conducted for $24 \mathrm{~h}$ at $25^{\circ} \mathrm{C}$.

The cells were washed, resuspended in lysis buffer $(20 \mathrm{mM}$ Tris- $\mathrm{HCl} \mathrm{pH}=7.5$, $100 \mathrm{mM} \mathrm{NaCl}, 5 \%$ glycerol, $2 \mathrm{mM} \mathrm{BME,} 5 \mathrm{mM}$ EDTA), and disrupted by highpressure homogenization (Xpress). Next, the broken cells were resuspended in lysis buffer at $50 \mathrm{mg} / \mathrm{mL}$. Unbroken cells and cell debris were removed by centrifugation at $4000 \times \mathrm{g}$ for $10 \mathrm{~min}$ and crude membranes were isolated by $90 \mathrm{~min}$ centrifugation at $165,000 \times g$. The membrane pellets were solubilized using solubilization buffer ( $50 \mathrm{mM}$ Tris- $\mathrm{HCl} \mathrm{pH}=7.5,150 \mathrm{mM} \mathrm{NaCl}, 20 \%$ (v/v) glycerol, $2 \mathrm{mM} \mathrm{BME}$ ) supplied with $2 \%(\mathrm{w} / \mathrm{v}) \mathrm{N}$-dodecyl $\beta$-d-maltoside (DDM), $0.2 \%(\mathrm{w} / \mathrm{v})$ cholesterol hemisuccinate (CHS) and EDTA-free protease inhibitor cocktail to a final concentration of $50 \mathrm{mg} / \mathrm{mL}$ for $2 \mathrm{~h}$. Insolubilized material was removed through 30 min centrifugation at 190,000×g. The supernatant was diluted 5 times in buffer
A (25 mM Tris- $\mathrm{HCl} \mathrm{pH}=7.5,150 \mathrm{mM} \mathrm{NaCl}, 10 \%$ (v/v) glycerol, $2 \mathrm{mM} \mathrm{BME})$ and loaded on a prepacked Sepharose $5 \mathrm{~mL}$ Histrap column equilibrated in $30 \mathrm{~mL}$ buffer A containing $0.05 \%(\mathrm{w} / \mathrm{v}) \mathrm{DDM}$ and $0.005 \%(\mathrm{w} / \mathrm{v})$ CHS. Three washing steps were employed to remove unspecifically bound proteins, i.e., $30 \mathrm{~mL}$ of buffer A with $10 \mathrm{mM}$ (final) imidazole, $0.1 \%$ (w/v) DDM and 0.01\% (w/v) CHS; then $20 \mathrm{mM}$ imidazole, $0.075 \%(\mathrm{w} / \mathrm{v}) \mathrm{DDM}$ and $0.0075 \%(\mathrm{w} / \mathrm{v}) \mathrm{CHS}$; and finally $40 \mathrm{mM}$ imidazole, $0.05 \%(\mathrm{w} / \mathrm{v}) \mathrm{DDM}$ and $0.005 \%(\mathrm{w} / \mathrm{v})$ CHS. Bound protein was eluted in buffer A with $0.05 \%$ (w/v) DDM and $0.005 \%$ (w/v) CHS and $300 \mathrm{mM}$ imidazole, using a one-step procedure. The eluate was assessed by sodium dodecyl sulfate-polyacrylamide gel electrophoresis (SDS-PAGE) and the fractions containing Ypk9 pooled and concentrated using Ultra-15 centrifugal concentrators (Amicon) with $100 \mathrm{kDa}$ MW cut-off. The sample was then applied to a Superose6 size exclusion chromatography column (Cytiva) equilibrated in buffer A with $0.05 \%(w / v)$ DDM and $0.005 \%(w / v)$ CHS. The peak fractions were pooled and concentrated to $5 \mathrm{mg} / \mathrm{mL}$ for the Nanodisc reconstitution or stored at $-80{ }^{\circ} \mathrm{C}$ for further application. The protein purity was assessed using SDS-PAGE.

MSP1E3D1 production and purification. The MSP1E3D $1^{33}$ plasmid was transformed into BL21(DE3) cells. Single colonies were incubated in $10 \mathrm{~mL}$ LB medium supplied with $25 \mathrm{mg} / \mathrm{L}$ kanamycin and cultivated $16 \mathrm{~h}$ at $37^{\circ} \mathrm{C}$ with shaking $200 \mathrm{rpm}$. The pre-culture was transferred into $1 \mathrm{~L} \mathrm{LB}$ medium with $25 \mathrm{mg} / \mathrm{L}$ kanamycin and incubated for $3 \mathrm{~h}$ at $37^{\circ} \mathrm{C}$ with shaking at $200 \mathrm{rpm}$. Protein production was induced through the addition of $1 \mathrm{mM}$ IPTG at an OD600 of 0.6 , and then continued at $37^{\circ} \mathrm{C}$ for $1 \mathrm{~h}$ following $3 \mathrm{~h}$ at $30^{\circ} \mathrm{C}$. The cell pellets were washed with PBS buffer and then re-suspended in PBS buffer supplied with $2 \mathrm{mM} \mathrm{MgCl}_{2}$ $0.01 \mathrm{mg} / \mathrm{mL}$ DNaseI and EDTA-free protease inhibitor cocktail $(50 \mathrm{mg} / \mathrm{mL})$. The cells were disrupted by $20 \mathrm{~min}$ sonication. Unbroken cells and cell debris were removed by centrifugation at $75,000 \times \mathrm{g}$ for $1 \mathrm{~h}$. The supernatant was filtered $(0.45 \mu \mathrm{m})$ and applied to Ni-NTA resin (Cytiva) for affinity purification. The column was then washed with $100 \mathrm{~mL}$ of the following buffers: (1) $40 \mathrm{mM}$ Tris$\mathrm{HCl} \mathrm{pH}=8.0,300 \mathrm{mM} \mathrm{NaCl}$ and $1 \%(\mathrm{v} / \mathrm{v})$ Triton; 2) $40 \mathrm{mM}$ Tris- $\mathrm{HCl} \mathrm{pH}=8.0$, $300 \mathrm{mM} \mathrm{NaCl}, 50 \mathrm{mM} \mathrm{Na}$-Cholate and $20 \mathrm{mM}$ Imidazole; 3) $100 \mathrm{ml} 40 \mathrm{mM}$ Tris$\mathrm{HCl} \mathrm{pH}=8.0,300 \mathrm{mM} \mathrm{NaCl}$ and $40 \mathrm{mM}$ Imidazole. The protein was eluted in $15 \mathrm{~mL}$ elution buffer $(40 \mathrm{mM}$ Tris- $\mathrm{HCl} \mathrm{pH}=8.0,300 \mathrm{mM} \mathrm{NaCl}$, and $400 \mathrm{mM}$ Imidazole). TEV protease was added to cleave the His-tag, and the sample was dialyzed for $16 \mathrm{~h}$ against $20 \mathrm{mM}$ Tris- $\mathrm{HCl} \mathrm{pH}=7.4,100 \mathrm{mM} \mathrm{NaCl}$ and $0.5 \mathrm{mM}$ EDTA. To separate the cleaved target protein from TEV protease, the sample was again applied to Ni-NTA resin, the flow-through collected and concentrated to $5 \mathrm{mg} / \mathrm{mL}$, flash-frozen in liquid nitrogen, and stored at $-80^{\circ} \mathrm{C}$ until further usage.

Nanodisc reconstitution. Yeast polar lipids dissolved in chloroform (Avanti Lipids, $25 \mathrm{mg} / \mathrm{mL}$ ) were gently dried under nitrogen gas and dissolved in $20 \mathrm{mM}$ Tris- $\mathrm{HCl} \mathrm{pH}=7.5,100 \mathrm{mM} \mathrm{NaCl}, 0.5 \%$ (w/v) DDM. The E2.P $\mathrm{S}_{\mathrm{i}}^{\mathrm{SPM}}$ structure was determined using protein reconstituted into MSP1D1 with a molar ratio of Ypk9: MSP1D1:lipids of 1:4:100. All the other structures were generated in the presence of MSP1E3D1, reconstituted with a molar ratio of Ypk9:MSP1D1:lipids of 1:10:300. The lipids were added to the protein sample, gently mixed, and incubated for $30 \mathrm{~min}$, and then the MSP protein and buffer added. $200 \mathrm{mg}$ bio-beads SM2 (Bio$\mathrm{Rad})$ were supplemented, and the sample was incubated for $2 \mathrm{~h}$ to remove detergents. The protein nanodisc complex was further purified by IMAC and sizeexclusion- chromatography using a Superdex 200 column (Cytiva). The Ypk9 nanodisc peak fractions were pooled and concentrated to $1 \mathrm{mg} / \mathrm{mL}$ for the cryo-EM sample preparation.

Cryo-EM sample preparation. Purified Ypk9 in nanodiscs was frozen at a concentration of $0.8-1.2 \mathrm{mg} / \mathrm{mL}$. Quantifoil $1.2 / 1.3$ holy carbon grids were glowdischarged using a Leica Coater ACE 200 for $40 \mathrm{~s}$ with $10 \mathrm{~mA}$ current. The grids were prepared using a Vitrobot Mark IV operated at $100 \%$ humidity and $4{ }^{\circ} \mathrm{C}$. In total, $3 \mu \mathrm{L}$ of purified protein was applied to each grid, incubated for $5 \mathrm{~s}$, blotted for $3 \mathrm{~s}$, and then plunge frozen into liquid ethane. Frozen grids were stored in liquid nitrogen until data collection. The different protein conformations were stabilized using supplements added immediately before freezing: E2Pinhib $(1 \mathrm{mM}$ $\left.\mathrm{BeF}_{3}{ }^{-}\right)$; E2P* ( $1 \mathrm{mM} \mathrm{SPM}$ and $1 \mathrm{mM} \mathrm{BeF}_{3}{ }^{-}$); E2.P. $\mathrm{P}_{\mathrm{i}}^{\mathrm{ALF} / \mathrm{SPM}}$ (1 mM SPM and $1 \mathrm{mM}$ $\mathrm{AlF}) ; \mathrm{E} 2 . \mathrm{P}_{\mathrm{i}}^{\mathrm{SPM}}(1 \mathrm{mM} \mathrm{SPM}$ and $1 \mathrm{mM} \mathrm{MgCl}$ ). All SPM containing samples were first incubated for $1 \mathrm{~h}$ at $18{ }^{\circ} \mathrm{C}$ and then heat-activated in a $40^{\circ} \mathrm{C}$ water bath for 5 min immediately before freezing. The E2Pinhib sample was only incubated at $18^{\circ} \mathrm{C}$ without heat treatment.

Cryo-EM data collection and data processing. The cryo-EM datasets were collected on two separate Titan Krios electron microscopes (FEI) operated at $300 \mathrm{kV}$ with either a Falcon3 detector in counting mode or a Gatan K3 detector in superresolution mode. For the Falcon 3 dataset, the pixel size was set to $0.832 \AA$ and the total dose was 40 e/Å2 in 40 frames. For the Gatan K3 datasets, the pixel size was $0.54295 \AA$ and the total dose was $50 \mathrm{e} / \mathrm{A} 2$ in 40 frames. An energy filter at $20 \mathrm{eV}$ was applied for the Gatan K3 data collection. All data were processed using cryosparc $^{34}$ following the procedures outlined in Supplementary Figs. 12-15 and Supplementary Table 1. For the Falcon3 dataset, the data were initially processed using full-frame motion correction and patch CTF determination. Blob particles with a diameter of $80-120 \AA$ were picked without templates and extracted to a box 
size of 360 pixels ( $300 \AA$ diameter) using local motion correction with doseweighting. Extracted particles were subjected to several rounds of reference-free two-dimensional class averaging to remove obvious junk, contamination, and empty nanodiscs. The cleaned particle set was processed following standard cryosparc workflow steps including ab-initial model reconstitution, multiple rounds of heterogeneous refinement, and non-uniform refinement iterations ${ }^{35}$. For the Gatan K3 dataset, the data were first motion-corrected using patch motion correction with $2 \times$ binning to micrographs with a pixel size of $1.086 \AA$. CTF parameters were determined using patch CTF. Blob particles were picked and extracted to a box size of 288 pixels ( $312 \AA$ diameter). After similar data processing steps as described for the Falcon3 datasets, the last particle set was re-extracted using local motion correction to super-resolution with a box size of 600 pixels to push the resolution of the final map.

Model building. The initial models were generated by the SWISS-MODEL online server ${ }^{36}$ using the corresponding sequences of Ypk9 and the EM structures of Spf1 (PDB-ID 6XMP and 6XMT) as templates. The missing residues were modeled as unstructured loops. The full-length models were then fitted into the corresponding cryo-EM density maps using the molecular dynamics flexible fitting (MDFF) method in an implicit solvent with secondary structure, cis-peptide, and chirality restraints to prevent overfitting ${ }^{37}$. The E2Pinhib model was then further refined by multiple rounds of manual adjustments in $\mathrm{Coot}^{38}$ and using MDFF and phenix_real_space_refine ${ }^{39}$. The dipping region of the NTD domain was built manually in Coot guided by side chain features in the density. The autoinhibitory tail was assigned manually based on density features. The other structures were generated using the same strategy, based on the first-built E2Pinhib structure. MolProbity implemented in Phenix was used for model validation ${ }^{40}$.

Figure preparation. All figures were generated using UCSF Chimera ${ }^{41}$ and ChimeraX ${ }^{42}$. Alignments were performed using Clustal Omega ${ }^{43}$ and visualized using ESPript 3.044.

ATPase activity. ATP turnover stimulated release of inorganic phosphate was performed using a commercial ATPase/GTPase Activity Assay Kit (MAK113 from Sigma). Absorbance was measured at $620 \mathrm{~nm}$ detecting liberated phosphate colored by malachite green. $40 \mathrm{ng}$ purified Ypk9 in DDM was used for $50 \mu \mathrm{L}$ final reaction mixture with assay buffer ( $50 \mathrm{mM}$ MOPS- $\mathrm{KOH} \mathrm{pH}=7.0,100 \mathrm{mM} \mathrm{KCl}, 5 \mathrm{mM}$ $\mathrm{MgCl}_{2}, 2 \mathrm{mM}$ 1,4-Dithiothreitol, $0.05 \%$ (w/v) DDM, 0.005\% (w/v) CHS). If present, the samples also contained final concentrations with $6 \mathrm{mM} \mathrm{SPM} 1 \mathrm{mM} \mathrm{BeF}_{3}{ }^{-}$and $1 \mathrm{mM} \mathrm{NaVO}_{4}$. The reactions were initiated through supplementation of $1 \mathrm{mM}$ ATP and performed at $37^{\circ} \mathrm{C}$ for $20 \mathrm{~min}$. Next, $20 \mu \mathrm{L}$ of the samples were transferred to a 384-well microplate, mixed with $80 \mu \mathrm{L}$ reagent, and incubated for an additional $15 \mathrm{~min}$ at $18{ }^{\circ} \mathrm{C}$. The measurements of absorbance at $620 \mathrm{~nm}$ were carried out using a Multiskan Sky Microplate Spectrophotometer (Thermo Scientific). The data in Fig. 2A are shown as three independent measurements based on one purification for each Ypk9 form, and the error bars represent the mean with $\mathrm{SD}$. The analysis of the SPM transport assay was performed using Graph Pad Prism 9.

Reporting summary. Further information on research design is available in the Nature Research Reporting Summary linked to this article.

\section{Data availability}

All data and materials supporting the findings in the paper are available from the corresponding author upon reasonable request. The structural coordinates and EM data have been deposited in the Protein Data Bank and in the Electron Microscopy Data Bank with the following accession numbers: E2Pinhib $(\mathrm{P} 8, \mathrm{E} 4), \mathrm{E} 2 \mathrm{P}^{*}(\mathrm{P} 5, \mathrm{E} 3), \mathrm{E} 2 \mathrm{P}_{\mathrm{i}}{ }_{\mathrm{A}}^{\mathrm{AlF} / \mathrm{SPM}}$ $(\mathrm{P} 1, \mathrm{E} 1)$, and E2.P $\mathrm{SPM}(\mathrm{P} 3, \mathrm{E} 2)$. Source data are provided with this paper.

Received: 11 March 2021; Accepted: 3 June 2021; Published online: 25 June 2021

\section{References}

1. Palmgren, M. G. \& Nissen, P. P-type ATPases. Annu. Rev. Biophys. 40, 243-266 (2011).

2. Kühlbrandt, W. Biology, structure and mechanism of P-type ATPases. Nat. Rev. Mol. Cell Biol. 5, 282-295 (2004).

3. Møller, A. B., Asp, T., Holm, P. B. \& Palmgren, M. G. Phylogenetic analysis of P5 P-type ATPases, a eukaryotic lineage of secretory pathway pumps. Mol. Phylogenetics Evolut. 46, 619-634 (2008).

4. McKenna, M. J. et al. The endoplasmic reticulum P5A-ATPase is a transmembrane helix dislocase. Science 369, eabc5809 (2020).

5. Sørensen, D. M. et al. Parkinson disease related ATP13A2 evolved early in animal evolution. PLoS ONE 13, e0193228 (2018).
6. Schröder, B. et al. Integral and associated lysosomal membrane proteins Traffic 8, 1676-1686 (2007).

7. Ugolino, J., Fang, S., Kubisch, C. \& Monteiro, M. J. Mutant Atp13a2 proteins involved in parkinsonism are degraded by ER-associated degradation and sensitize cells to ER-stress induced cell death. Hum. Mol. Genet. 20, 3565-3577 (2011).

8. Dehay, B. et al. Loss of P-type ATPase ATP13A2/PARK9 function induces general lysosomal deficiency and leads to Parkinson disease neurodegeneration. Proc. Natl Acad. Sci. USA 109, 9611-9616 (2012).

9. Ramirez, A. et al. Hereditary parkinsonism with dementia is caused by mutations in ATP13A2, encoding a lysosomal type 5 P-type ATPase. Nat. Genet. 38, 1184-1191 (2006).

10. Park, J.-S., Blair, N. F. \& Sue, C. M. The role of ATP13A2 in Parkinson's disease: clinical phenotypes and molecular mechanisms: ATP13A2 in Parkinson's Disease. Mov. Disord. 30, 770-779 (2015).

11. Gitler, A. D. et al. $\alpha$-Synuclein is part of a diverse and highly conserved interaction network that includes PARK9 and manganese toxicity. Nat. Genet. 41, 308-315 (2009).

12. van Veen, S. et al. ATP13A2 deficiency disrupts lysosomal polyamine export. Nature 578, 419-424 (2020).

13. Sørensen, D. M., Buch-Pedersen, M. J. \& Palmgren, M. G. Structural divergence between the two subgroups of P5 ATPases. Biochimica et Biophysica Acta (BBA). Bioenergetics 1797, 846-855 (2010).

14. Holemans, T. et al. A lipid switch unlocks Parkinson's disease-associated ATP13A2. Proc. Natl Acad. Sci. USA 112, 9040-9045 (2015).

15. Post, R. L., Hegyvary, C. \& Kume, S. Activation by adenosine triphosphate in the phosphorylation kinetics of sodium and potassium ion transport adenosine triphosphatase. J. Biol. Chem. 247, 6530-6540 (1972).

16. Albers, R. W. Biochemical aspects of active transport. Annu. Rev. Biochem. 36, 727-756 (1967).

17. Danko, S. J. \& Suzuki, H. The Use of Metal Fluoride Compounds as Phosphate Analogs for Understanding the Structural Mechanism in P-type ATPases. in (ed Bublitz, M.) P-Type ATPases vol. 1377 195-209 (Springer New York, 2016).

18. Hiraizumi, M., Yamashita, K., Nishizawa, T. \& Nureki, O. Cryo-EM structures capture the transport cycle of the P4-ATPase flippase. Science 365, 1149-1155 (2019).

19. Olesen, C. Dephosphorylation of the calcium pump coupled to counterion occlusion. Science 306, 2251-2255 (2004).

20. Nakanishi, H. et al. Transport cycle of plasma membrane flippase ATP11C by Cryo-EM. Cell Rep. 32, 108208 (2020).

21. Nakanishi, H. et al. Crystal structure of a human plasma membrane phospholipid flippase. J. Biol. Chem. 295, 10180-10194 (2020).

22. Sorensen, T. L.-M. Phosphoryl transfer and calcium ion occlusion in the calcium pump. Science 304, 1672-1675 (2004)

23. Ekberg, K., Palmgren, M. G., Veierskov, B. \& Buch-Pedersen, M. J. A novel mechanism of P-type ATPase autoinhibition involving both termini of the protein. J. Biol. Chem. 285, 7344-7350 (2010).

24. Bækgaard, L., Fuglsang, A. T. \& Palmgren, M. G. Regulation of plant plasma membrane $\mathrm{H}+$ - and $\mathrm{Ca} 2+-\mathrm{ATPases}$ by terminal domains. J. Bioenerg. Biomembr. 37, 369-374 (2005).

25. Wu, C.-C., Rice, W. J. \& Stokes, D. L. Structure of a copper pump suggests a regulatory role for its metal-binding domain. Structure 16, 976-985 (2008).

26. Jacquot, A. et al. Phosphatidylserine stimulation of Drs2p.Cdc50p lipid translocase dephosphorylation is controlled by phosphatidylinositol-4phosphate. J. Biol. Chem. 287, 13249-13261 (2012).

27. Timcenko, M. et al. Structure and autoregulation of a P4-ATPase lipid flippase. Nature 571, 366-370 (2019).

28. Natarajan, P. et al. Regulation of a Golgi flippase by phosphoinositides and an ArfGEF. Nat. Cell Biol. 11, 1421-1426 (2009).

29. Chantalat, $S$. The Arf activator Gea2p and the P-type ATPase Drs2p interact at the Golgi in Saccharomyces cerevisiae. J. Cell Sci. 117, 711-722 (2004).

30. Chalat, M., Moleschi, K. \& Molday, R. S. C-terminus of the P4-ATPase ATP8A2 functions in protein folding and regulation of phospholipid flippase activity. MBoC 28, 452-462 (2017).

31. Scharff-Poulsen, P. \& Pedersen, P. A. Saccharomyces cerevisiae-based platform for rapid production and evaluation of eukaryotic nutrient transporters and transceptors for biochemical studies and crystallography. PLoS ONE 8, e76851 (2013).

32. Tripp, J. D., Lilley, J. L., Wood, W. N. \& Lewis, L. K. Enhancement of plasmid DNA transformation efficiencies in early stationary-phase yeast cell cultures: enhancement of DNA transformation in yeast cells. Yeast 30, 191-200 (2013).

33. Ritchie, T. K. et al. Reconstitution of membrane proteins in phospholipid bilayer nanodiscs. in Methods in Enzymology vol. 464 211-231 (Elsevier, 2009).

34. Punjani, A., Rubinstein, J. L., Fleet, D. J. \& Brubaker, M. A. cryoSPARC: algorithms for rapid unsupervised cryo-EM structure determination. Nat. Methods 14, 290-296 (2017). 
35. Punjani, A., Zhang, H. \& Fleet, D. J. Non-uniform refinement: adaptive regularization improves single-particle cryo-EM reconstruction. Nat. Methods 17, 1214-1221 (2020).

36. Waterhouse, A. et al. SWISS-MODEL: homology modelling of protein structures and complexes. Nucleic Acids Res. 46, W296-W303 (2018).

37. Trabuco, L. G., Villa, E., Mitra, K., Frank, J. \& Schulten, K. Flexible fitting of atomic structures into electron microscopy maps using molecular dynamics. Structure 16, 673-683 (2008).

38. Emsley, P., Lohkamp, B., Scott, W. G. \& Cowtan, K. Features and development of Coot. Acta Crystallogr. D Biol. Crystallogr. 66, 486-501 (2010).

39. Afonine, P. V. et al. Real-space refinement in PHENIX for cryo-EM and crystallography. Acta Crystallogr. D Struct. Biol. 74, 531-544 (2018).

40. Chen, V. B. et al. MolProbity: all-atom structure validation for macromolecular crystallography. Acta Crystallogr. D Biol. Crystallogr. 66, 12-21 (2010).

41. Pettersen, E. F. et al. UCSF Chimera: a visualization system for exploratory research and analysis. J. Comput. Chem. 25, 1605-1612 (2004).

42. Pettersen, E. F. et al. UCSF ChimeraX: structure visualization for researchers, educators, and developers. Protein Sci. 30, 70-82 (2021).

43. Madeira, F. et al. The EMBL-EBI search and sequence analysis tools APIs in 2019. Nucleic Acids Res. 47, W636-W641 (2019).

44. Robert, X. \& Gouet, P. Deciphering key features in protein structures with the new ENDscript server. Nucleic Acids Res. 42, W320-W324 (2014).

45. Pei, J. \& Grishin, N. V. AL2CO: calculation of positional conservation in a protein sequence alignment. Bioinformatics 17, 700-712 (2001).

\section{Acknowledgements}

We would like to thank Julian Conrad and Marta Carroni at SciLifeLab Stockholm as well as Tillmann Hanns Pape at CFIM University of Copenhagen for assistance with the Cryo-EM data collection. We acknowledge access to the computational resources from the Danish National Supercomputer for Life Sciences (Computerome). CG is currently paid by The BRIDGE-Translational Excellence Program at the University of Copenhagen funded by the Novo Nordisk Foundation (NNF18SA0034956). KW is supported by the Lundbeck Foundation. PG is supported by the following Foundations: Lundbeck, Knut and Alice Wallenberg, Carlsberg, Novo-Nordisk, Brødrene Hartmann, Agnes og Poul Friis, Augustinus, Crafoord as well as The Per-Eric and Ulla Schyberg. Funding is also obtained from The Independent Research Fund Denmark, the Swedish Research Council, and through a Michaelsen scholarship. The funders had no role in study design, data collection, and analysis, decision to publish, or preparation of the paper.

\section{Author contributions}

P.L. performed cloning, overproduction, purification, and nanodisc-reconstitution for the structural and functional studies. Cryo-EM sample preparation and data analysis were primarily performed by K.W.. P.L. and K.W. assisted with Cryo-EM data collections at
SciLifeLab Stockholm, and K.W. executed the data collection at CFIM University of Copenhagen. P.L., N.S., C.G., and K.W. built and refined the structures. N.S. prepared the figures except for Supplementary Figs. 12-15 that were generated by K.W. P.L., K.W., and P.G. contributed to the identification of the scientific problem and experimental planning. P.L., N.S., C.G., K.W., and P.G. conducted data analysis and interpretation. N.S. and P.G. wrote the first draft. All authors commented on the paper. P.G. supervised the project.

\section{Funding}

Open access funding provided by Lund University.

\section{Competing interests}

The authors declare no competing interests.

\section{Additional information}

Supplementary information The online version contains supplementary material available at https://doi.org/10.1038/s41467-021-24148-y.

Correspondence and requests for materials should be addressed to P.G.

Peer review information Nature Communications thanks Kazuhiro Abe and Hugo Adamo for their contribution to the peer review of this work. Peer reviewer reports are available.

Reprints and permission information is available at http://www.nature.com/reprints

Publisher's note Springer Nature remains neutral with regard to jurisdictional claims in published maps and institutional affiliations.

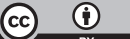

Open Access This article is licensed under a Creative Commons Attribution 4.0 International License, which permits use, sharing, adaptation, distribution and reproduction in any medium or format, as long as you give appropriate credit to the original author(s) and the source, provide a link to the Creative Commons license, and indicate if changes were made. The images or other third party material in this article are included in the article's Creative Commons license, unless indicated otherwise in a credit line to the material. If material is not included in the article's Creative Commons license and your intended use is not permitted by statutory regulation or exceeds the permitted use, you will need to obtain permission directly from the copyright holder. To view a copy of this license, visit http://creativecommons.org/ licenses/by/4.0/.

(C) The Author(s) 2021 